Arq. Bras. Med. Vet. Zootec., v.56, n.1, p.119-122, 2004

\title{
Comunicação
}

[Communication]

\section{Aspectos clínicos e de laboratório de cães soropositivos para leishmaniose}

[Clinical and laboratorial aspects of seropositive dogs to leishmaniosis]

\author{
D.G. Mattos Jr. ${ }^{1}$, J.M. Pinheiro ${ }^{2}$, R.C. Menezes ${ }^{1}$, D.A. Costa $^{3}$ \\ ${ }^{1}$ Faculdade de Veterinária da Universidade Federal Fluminense \\ Departamento de Saúde Coletiva Veterinária e Saúde Pública - MSV \\ Rua Dr. Vital Brazil Filho, 64 \\ 24230-260 - Niterói, RJ \\ ${ }^{2}$ Centro de Controle de Zoonoses do Município de Niterói, RJ \\ ${ }^{3}$ Mestranda em Medicina Veterinária da FVUFF - Niterói, RJ
}

A leishmaniose é uma doença parasitária cujos agentes etiológicos são diferentes espécies de protozoários do gênero Leishmania, que são transmitidos pela picada de dípteros da família Psychodidae, dos gêneros Lutzomyia e Phlebotomus.

Dentre as leishmanioses, o tipo visceral é a forma mais grave e é considerada uma zoonose que tem o cão como principal reservatório no ciclo peri-doméstico. Segundo Marzochi et al. (1985), sua ocorrência tem sido registrada em diversos estados do Brasil e também na área do Grande Rio, em cães provenientes de regiões de matas, encostas e aglomerados ainda não urbanizados. Segundo os autores, a freqüência de cães infectados assintomáticos é de 63,2\%.

Diversas regiões do município de Niterói oferecem condições ecológicas adequadas à multiplicação dos vetores e conseqüente transmissão da leishmaniose aos cães vadios, constituindo assim grande problema de saúde pública. Até o momento, pouco se conhece sobre os aspectos clínicos dessa doença nos cães de rua deste município.

O objetivo do trabalho foi estudar os aspectos clínicos e laboratoriais da doença visando avaliar a importância dessas alterações em cães da região.

A partir de exames sorológicos de imunofluorescência indireta realizados no Instituto Jorge Vaitsman, Rio de Janeiro, 18 cães apreendidos nas ruas e recolhidos ao Centro de Controle de Zoonoses do Município de Niterói (CECOZ) foram divididos em dois grupos de nove soropositivos para Leishmania (grupo 1) e nove soronegativos (grupo 2). Foram realizados exames clínicos e procederam-se três colheitas de sangue em intervalos de 15 dias (dia 0 na confirmação sorológica, dia 15 e dia 30 após a primeira colheita). As análises laboratoriais foram realizadas conforme Coles (1986).

Os cães, isolados no canil do CECOZ, receberam ração e água ad libidum.

Os resultados da freqüência dos sinais clínicos estão na Tab.1 Observou-se maior freqüência deles nos cães soropositivos, sendo linfadenopatia, úlceras de pele e hipertermia os sinais clínicos mais observados.

Os valores médios do eritrograma, leucograma e bioquímicos séricos são mostrados nas Tab. 2, 3 e 4 , respectivamente.

Trabalho financiado pela FAPERJ - PROJETO PAI - E-26/170.681/2001

Recebido para publicação, após modificações, em 4 de abril de 2003

E-mail: dgmjrvet@vm.uff.br 
Tabela 1. Freqüência de sinais clínicos observados em cães soropositivos e soronegativos para Leishmania, no período de 30 dias em Niterói, RJ

\begin{tabular}{lcccc}
\hline \multirow{2}{*}{ Sinal clínico } & \multicolumn{2}{c}{ Soropositivo } & \multicolumn{2}{c}{ Soronegativo } \\
\cline { 2 - 5 } & $\mathrm{n}$ & $\%$ & $\mathrm{n}$ & $\%$ \\
\hline Com mais de um sinal & 8 & 88,8 & 3 & 33,3 \\
clínico & 6 & 66,6 & 0 & 0 \\
Linfadenopatia & 5 & 55,5 & 7 & 77,7 \\
Lesões por ectoparasitos & & 33,3 & 0 & 0 \\
- carrapatos & 3 & 33,3 & 2 & 22,2 \\
Úlceras de pele & 3 & 22,2 & 1 & 11,1 \\
Hipertermia & 2 & 22,2 & 2 & 22,2 \\
Apatia & 2 & 22,2 & 0 & 0 \\
Caquexia & 2 & 22,2 & 0 & 0 \\
Ulceração de mucosas & 2 & 22,2 & 3 & 33,3 \\
Descamação e eczema & 2 & 22,2 & 0 & 0 \\
Alopecia & 2 & 22,2 & 0 & 0 \\
Cicatriz de pele & 2 & 22,1 & 0 & 0 \\
Onicogrifose & 1 & 11,1 \\
Vômito & 1 & 11,1 & 2 & 22,2 \\
\hline Enterorragia & &
\end{tabular}

$\mathrm{n}=18 \mathrm{em}$ soropositivos e soronegativos.

Tabela 2. Valores médios do eritrograma de cães soropositivos e soronegativos para Leishmania em três colheitas de sangue, no período de 30 dias em Niterói, RJ

\begin{tabular}{lcc}
\hline Exame & Soropositivo & Soronegativo \\
\hline Hematimetria & $4.508 .000 \mathrm{~mm}^{3}$ & $5.300 .000 \mathrm{~mm}^{3}$ \\
Hemoglobina & $10,4 \mathrm{~g} \%$ & $13,5 \mathrm{~g} \%$ \\
Volume globular & $31,5 \%$ & $37,5 \%$ \\
VGH & $65,5 \mathrm{fl}$ & $65,5 \mathrm{fl}$ \\
$\mathrm{CHM}$ & $33,2 \%$ & $32,2 \%$ \\
\hline
\end{tabular}

$\mathrm{n}=18$ em soropositivos e soronegativos

Tabela 3. Valores médios do leucograma de cães soropositivos e soronegativos para Leihsmania em três colheitas de sangue, no período de 30 dias em Niterói, RJ

\begin{tabular}{lcc}
\hline Exame & Soropositivo & Soronegativo \\
\hline Leucometria global & $20.925 \mathrm{~mm}^{3}$ & $1.200 \mathrm{~mm}^{3}$ \\
Basófilos & 525 & 105 \\
Bastonetes & 1.566 & 110,5 \\
Segmentados & 15.007 & 7.000 \\
Linfócitos & 3.290 & 3.713 \\
Monócitos & 1.560 & 775 \\
Eosinófilos & 2.490 & 780 \\
\hline
\end{tabular}

$\mathrm{n}=18$ em soropositivos e soronegativos.
Tabela 4. Valores médios bioquímicos séricos de cães soropositivos e soronegativos para Leihsmania em três colheitas de sangue, no período de 30 dias em Niterói, RJ

\begin{tabular}{lcc}
\hline Constituinte & Soropositivo & Soronegativo \\
\hline Proteínas totais & $7,3 \mathrm{~g} / \mathrm{dl}$ & $6,95 \mathrm{~g} / \mathrm{dl}$ \\
Albumina & $3,7 \mathrm{~g} / \mathrm{dl}$ & $3,55 \mathrm{~g} / \mathrm{dl}$ \\
Globulina & $3,8 \mathrm{~g} / \mathrm{dl}$ & $3,4 \mathrm{~g} / \mathrm{dl}$ \\
Uréia & $41,5 \mathrm{mg} / \mathrm{dl}$ & $32,5 \mathrm{mg} / \mathrm{dl}$ \\
Ácido úrico & $2,2 \mathrm{mg} / \mathrm{dl}$ & $1,2 \mathrm{mg} / \mathrm{dl}$ \\
Creatinina & $1,0 \mathrm{mg} / \mathrm{dl}$ & $1,4 \mathrm{mg} / \mathrm{dl}$ \\
Lipídios totais & $366,5 \mathrm{mg} / \mathrm{dl}$ & $308 \mathrm{mg} / \mathrm{dl}$ \\
Colesterol & $220 \mathrm{mg} / \mathrm{dl}$ & $216,5 \mathrm{mg} / \mathrm{dl}$ \\
Triglicerídeos & $136,3 \mathrm{mg} / \mathrm{dl}$ & $142,5 \mathrm{mg} / \mathrm{dl}$ \\
Glicose & $109,5 \mathrm{mg} / \mathrm{dl}$ & $88,5 \mathrm{mg} / \mathrm{dl}$ \\
Cálcio & $10,5 \mathrm{mg} / \mathrm{dl}$ & $11 \mathrm{mg} / \mathrm{dl}$ \\
Fósforo & $4,4 \mathrm{mg} / \mathrm{dl}$ & $4,05 \mathrm{mg} / \mathrm{dl}$ \\
\hline $\mathrm{n}=18$ em soropositivos & soronegativos.
\end{tabular}

$\mathrm{n}=18$ em soropositivos e soronegativos.

Keenan et al. (1984) mencionaram que a linfadenopatia, aliada à esplenomegalia, ocorre entre 27 e 55 dias pós-inoculação experimental de Leishmania infantum em cães. A linfadenopatia observada nos cães soropositivos também foi registrada por outros investigadores (Sciaramella et al., 1997). A observação de mais de um sinal clínico e a linfadenopatia como o dado mais freqüente, aliados aos achados epidemiológicos, podem levar à suspeita da doença, porém a confirmação do diagnóstico só deve ser feita por meio de exames sorológicos.

Marzochi et al. (1985) salientaram que o emagrecimento dos cães em áreas de leishmaniose visceral é freqüente. Isto não foi observado nesta pesquisa, fato atribuído ao curto período de estudo.

As lesões de pele foram semelhantes às relatadas por Jongstaffe et al. (1983), Marzochi et al. (1985) e Sciaramella et al. (1997), em cães soropositivos para leishmaniose.

Apenas dois cães soropositivos se apresentaram com onicogrifose, considerado um sinal patognomônico para leishmaniose visceral por Sciaramella et al. (1997) e Strauss-Ayali e Baneth (2001). Marzochi et al. (1985) explicaram que essa alteração é atribuída à baixa atividade dos cães e conseqüente falta de desgaste das unhas. 
Os valores médios do eritrograma dos cães soropositivos foi menor que os dos cães soronegativos. Há divergências na literatura quanto a esse tipo de resultado (Dedet, 1979; Ettinger, 1992; Nieto et al., 1997).

Observou-se monocitose nos cães soropositivos, resultado também descrito por Brandonisio et al. (1986) como sendo monocitose compensatória. Da mesma forma, a neutrofilia em cães com leishmaniose visceral também foi observada por Keenan et al. (1984) e Swenson et al. (1988).

Os cães soropositivos apresentaram valores bioquímicos séricos médios ligeiramente superiores aos dos cães soronegativos. Contudo, os níveis médios de cálcio dos soropositivos estavam dentro dos parâmetros normais segundo Coles (1986), e ligeriamente mais baixos do que os dos cães soronegativos. Estas observações confirmam as de Ramos et al. (1994) que encontraram níveis séricos de cálcio normais em 15 cães com leishmaniose visceral provenientes do Rio de Janeiro. Esses investigadores observaram que nos cães com leishmaniose as taxas de colesterol apresentaram valores acima da normal e as de triglicerídeos estavam dentro dos parâmetros normais segundo Coles (1986). No presente trabalho, as taxas de colesterol apresentaram-se altas nos dois grupos de cães. Isto pode ter ocorrido devido ao regime alimentar (ração balanceada) que pode ter interferido nas taxas de colesterol, conforme foi observado por Lane e Robinson (1970), que relataram o condicionamento dessas taxas à dieta. Também não pode ser descartada uma alteração pós-prandial.

Os valores médios séricos dos cães soropositivos apresentaram oscilação acima e abaixo dos valores médios apresentados pelos soronegativos. Medway et al. (1973) e Tryphonas et al. (1977) também registraram as mesmas oscilações para os níveis séricos de lipídeos totais, creatinina, ácido úrico, albumina e globulina de cães soropositivos para Leishmania. Ramos et al. (1994) revelaram que a exceção seria nos níveis de colesterol (valores máximos acima da normalidade ), triglicerídeos (dentro dos parâmetros normais), glicose ( valores mínimos abaixo dos parâmetros normais) e cálcio (valores máximos acima dos parâmetros normais).

Oscilações relacionadas aos níveis de constituintes séricos em cães com leishmaniose visceral foram registrados por Medway et al. (1973) e Tryphonas et al. (1977) e devem ser consideradas quando se estudam as infecções experimentais e crônicas por leishmaniose. Em termos práticos os constituintes séricos são desprovidos de valor para diagnóstico, pois a baixa especificidade dos exames hematológicos e bioquímicos séricos torna imprescindível o exame sorológico. Dessa forma, a conduta laboratorial proposta não forneceu subsídios para a conclusão de um diagnóstico compatível com leishmaniose em cães recém capturados e mantidos em observação por 30 dias.

Palavra-chave: cão, leishmaniose, aspectos clínicos, diagnóstico

\section{AGRADECIMENTOS}

À Dra. Nádia Almosny, professora do Departamento de Patologia e Clínica Veterinária e responsável pelo Laboratório de Patologia Clínica da Faculdade de Veterinária da Universidade Federal Fluminense e à Dra. Vanda C. Pandolpho, Médica Veterinária responsável pelo setor de Doenças Parasitárias do Instituto Municipal de Medicina Veterinária Jorge Vaitsman do Estado do Rio de Janeiro.

\begin{abstract}
Leihsmaniasis is a protozoal disease transmitted by dipteran insects. The dog is an important domestic host of both visceral and cutaneous forms of the disease. These forms affects humans in many countries, and it is considered a serious risk for human health. This study reports clinical and laboratorial data from 18 dogs. Nine dogs were positive by indirect immunofluorescence technique and the other nine were negative. The dogs were clinically monitored for 30 days and laboratorial changes were assessed. Three blood samples were collected for hemogram and serum biochemistry at 0, 15, and 30 days. The clinical signs of positive dogs were compatible with the disease, including lymphadenopathy, cutaneous ulcers,
\end{abstract}


hyperthermia, apathy, cachexia and ulcerated mucosa. Marked differences were observed in the blood cell counts and blood biochemistry between positive and negative dogs. In conclusion, blood and biochemistry analyses of positive dogs were not sufficient to diagnose the disease within the period studied. The serologic test associated with the clinical signs are important to confirm the diagnosis.

Keywords: dogs, Leishmaniasis, clinical signs, diagnostic

\section{REFERÊNCIAS BIBLIOGRÁFICAS}

BRANDONISIO, O.; CECI, L.; CEDOLA, M.C. Phagocytosis of Leishmania infantum promastigotes by monocytes isolated from leishmanias-infected dogs. Microbiologica, v.9, p.173-178, 1986.

COLES, E.H. Veterinary clinical pathology. Philadelphia: W.B. Saunders, 1986. 615p.

DEDET, J.P. Les leishmanioses em Afrique du Nord. Bull. Inst. Pasteur, n.77, p.49-82, 1979.

ETTINGER, S.J. Tratado de medicina interna veterinária: moléstias do cão e do gato. 3.ed. São Paulo: Editora Manole, 1992. 2557p. 4v.

JONGSTAFFE，J.A.; JEFFERIES，A.R.; KELLY, D.F. et al. Leishmanisis in imported dogs in the United Kingdom a potential human health. J. Small Anim. Pract., v.24, p.23-30, 1983.

KEENAN, C.M.; HENDRICKS, L.D.; LIGHTNER, L. et al. Visceral Leishmaniasis in the German Shepherd Dog. I. Infection, clinical disease and clinical pathology. Vet. Pathol., v.21, p.80-86, 1984.

LANE, D.R; ROBINSON, R. The utility of biochemical screening in dogs. Br. Vet. J., v.126, p.230, 1970.

MARZOCHI, M.C.A.; COUTINHO, S.G.; SOUZA, W.J.S. et al. Canine Visceral Leishmaniasis in Rio de Janeiro, Brazil. Clinical, parasitological, therapeutical and epidemiological findings (1977-1983). Mem. Inst. Oswaldo Cruz, v.80, p.349-357, 1985.
MEDWAY, W.; PRIER, J.E.; WILKINSON, J.S. Patologia clínica veterinária. México: Uteha, 1973. 532p.

NIETO, C.G.; MIRÓN, C.; GARCÍA-ALONSO, M. et al. Estudio de um caso de ascitis asociada a leishmaniosis canina. In: CONGRESSO IBÉRICO DE PARASITOLOGIA, 5.; CONGRESSO PORTUGUÊS DE PARASITOLOGIA, 3.; CONGRESSO ESPAÑOL DE PARASITOLOGIA, 10., 1997, Lisboa. Anais... Lisboa: Acta Parasitológica Portuguesa, v.4, n.1-2, p.133, 1997

RAMOS, G.P.; RANGEL, F.B.F.; BOTELHO, G.G. Valores bioquímicos séricos de cães portadores de leishmaniose visceral. Rev. Bras. Med. Vet., v.16, p.23-25, 1994.

SCIARAMELLA, P.; OLIVA, G.; DE LUNA, R. et al. retrospective clinical study of canine leishmaniasis in 150 dogs naturally infected by Leishmania infantum. Vet. Rec., v.141, p.539-543, 1997.

STRAUSS-AYALI, D.; BANETH, G. Canine visceral Leishmaniosis. In: CHARMICHAEL, L. Recent advances in canine infectious diseases. Ithaca: Int. Vet. Inf. Serv., 2001. p.1-14.

SWENSON, C.L.; SILVERMAN, J.; STROMBERG, P.C. et al. Visceral leishmaniasis in an English Foxhound from an Ohio research colony. J. Am. Vet. Med. Assoc., v.193, p.1089-1092, 1988.

TRYPHONAS, L.; ZAWIDSKA, Z.; BERNARD, M.A. et al. Visceral leishmaniasis in dog: clinical, hematological and pathological observations. Can. J. Comp. Med., v.41, p.1-12, 1977. 